\title{
Network Pharmacology Analysis of Traditional Chinese Medicine Formula Shuang Di Shou Zhen Tablets Treating Nonexudative Age-Related Macular Degeneration
}

\author{
Yue Fang $\mathbb{D}$, Xinquan Liu $(\mathbb{D}$, and Jing Su $\mathbb{D}$ \\ Department of Ophthalmology, Longhua Hospital Affiliated Shanghai University of Traditional Chinese Medicine, \\ Shanghai 200032, China \\ Correspondence should be addressed to Jing Su; sujinglucky@163.com
}

Received 4 January 2021; Revised 10 March 2021; Accepted 13 March 2021; Published 24 March 2021

Academic Editor: Kanwal Rehman

Copyright (c) 2021 Yue Fang et al. This is an open access article distributed under the Creative Commons Attribution License, which permits unrestricted use, distribution, and reproduction in any medium, provided the original work is properly cited.

Objective. To analyze the pharmacological mechanism of the treatment of dry age-related macular degeneration (dry AMD) based on a network pharmacological approach of Shuang Di Shou Zhen Tablets (SDSZT) and to provide a new reference for the current lack of effective treatment of dry AMD. Methods. The main chemical constituents and their targets of Rehmanniae Radix Praeparata, Ligustrum lucidum, Mori Fructus, Paeonia albiflora, Rhizoma Dioscoreae, Alisma orientale, Schisandra chinensis, Radix Polygoni Multiflori Preparata, Ophiopogon japonicus, and Radix Rehmanniae were obtained from the Traditional Chinese Medicine Systems Pharmacology Database and Analysis Platform (TCMSP) and Traditional Chinese Medicine Integrated Database (TCMID). The active ingredients of traditional Chinese medicine were screened according to Absorption, Distribution, Metabolism, and Excretion (ADME), the gene names of the targets of each active ingredient were obtained from the Uniprot database, the main targets of dry AMD were obtained from GeneCards and DisGeNET database, and the protein interaction analysis was performed on the String database. The Metascape database was used to analyze the "drug-component-target" and the biological processes and networks involved, and then, Cytoscape 3.8.1 was used to construct the "SDSZT component-dry AMD target-pathway" network. Results. The main active ingredients of SDSZT for dry AMD treatment are quercetin, kaempferol, luteolin, $\beta$-glutamine, $\beta$-carotene, etc. And, the core targets are RAC-alpha serine/threonine-protein kinase (AKT1), prostaglandin G/H synthase 1 (PTGS1), tumor necrosis factor (TNF), transcription factor AP-1 (JUN), apoptosis regulator Bcl-2 (BCL2), caspase-3 (CASP3), phosphatidylinositol 4,5-bisphosphate 3-kinase catalytic subunit gamma isoform (PIK3CG), androgen receptor (AR), apoptosis regulator BAX (BAX), etc. The biological pathways for the treatment of age-related macular degeneration by SDSZT mainly act on pathways in cancer, fluid shear stress and atherosclerosis, and TNF signaling pathway, and the main function of SDSZT is to regulate intracellular cytokine receptor binding. Conclusion. This study initially reveals the multiconstituent, multitarget, and multipathway mechanism of action of SDSZT in the treatment of dry AMD and provides the basis for the clinical application of SDSZT.

\section{Introduction}

Age-related macular degeneration (AMD) is one of the most common eye diseases in ophthalmology and is one of the leading causes of blindness in the elderly population [1]. It can be divided into nonexudative AMD (dry AMD) and neovascular AMD (wet AMD). Existing anti-vascular endothelial growth factor (anti-VEGF) is known to be effective in the treatment of wet AMD; however, there is a lack of definitive and effective therapeutic measures for dry AMD. In recent years, there have been numerous studies on the treatment of dry AMD, such as complement factors [2], antiinflammatory therapy [3], acupuncture [4], and traditional Chinese medicine $[5,6]$. However, none of these can be considered definitive and effective treatments.

The advantages of traditional medicine in treating AMD should not be overlooked. The treatment mechanism of traditional Chinese medicine is not only to a single target, 
but is also based on the holistic view of traditional Chinese medicine to provide a multitarget, multifaceted, and comprehensive treatment of the disease, with high safety. Network pharmacology is the use of known drug components, component targets, and known targets of disease to predict the likelihood of a drug's potential to treat a disease, and the network is able to comprehensively respond to possible mechanisms of drug interventions. Through multipathway modulation of signaling pathways, network pharmacology, as a new tool for drug research, can effectively tap into the active ingredients of traditional Chinese medicine, discard toxic ingredients, and treat the corresponding diseases with maximum stability and precision.

Shuang Di Shou Zhen Tablet (SDSZT) is a self-made ophthalmic medicine for Longhua Hospital, Shanghai. For many years, SDSZTs have been used in ophthalmology clinics to treat age-related eye diseases (deficiency of liveryin and kidney-yin type: The deficiency of liver and kidney in traditional Chinese medicine, which means patients with dim eyesight, metamorphosis, drusen, and pigmentary disturbance in macula, accompanied by general symptoms of dizziness, tinnitus, lassitude in loin and legs, blackish tongue with thin coating, and thready thin pulse). In preliminary clinical observations, it was found that treatment with vitamin $\mathrm{C}$ and vitamin $\mathrm{E}$ combined with SDSZT resulted in a smaller number of Amsler table deformation frames and a smaller extent of central visual field defects in patients with dry AMD compared to vitamin $\mathrm{C}$ and vitamin E alone [6, 7]. This formula contains 10 herbs: Rehmanniae Radix Praeparata, Ligustrum lucidum, Mori Fructus, Paeonia albiflora, Rhizoma Dioscoreae, Alisma orientale, Schisandra chinensis, Radix Polygoni Multiflori Preparata, Ophiopogon japonicus, and Radix Rehmanniae, of which Rehmanniae Radix Praeparata and Ligustrum lucidum are popular herbs for research on their antioxidant, anti-inflammatory, and lipid metabolizing components in recent years [8-12]. Therefore, this study, based on network pharmacology approach, explores the active ingredient targets of SDSZT and the molecular mechanism of its treatment for dry AMD disease and provides a certain theoretical basis for the follow-up study.

\section{Materials and Methods}

2.1. Potential Drug Targets Prediction for SDSZT. The websites of TCMSP (Traditional Chinese Medicine Systems Pharmacology Database and Analysis Platform) (http://lsp. nwu.edu.cn/tcmsp.php) [13] and TCMID (Traditional Chinese Medicine Integrated Database) (http://119.3.41.228: $8000 / \mathrm{tcmid} /$ ) [14] were used to query the chemical composition of $10 \mathrm{TCM}$ herbal ingredients, and ADME values of oral bioavailability $(\mathrm{OB}) \geq 30 \%$ and drug-likeness $(\mathrm{DL}) \geq 0.18$ [15-17] were set for the active ingredients. Preliminary screening was done to obtain active ingredients and targets for each active ingredient. After the screening, the gene names of the relative targets were obtained within the Uniprot protein database (http://www.uniprot.org) for specification. The drug-component-target intersection was mapped by Cytoscape 3.7.1 software [18].
2.2. Collection of Known AMD-Related Targets. Using "dry age-related macular degeneration" and "nonexecutive agerelated macular degeneration" as keywords, we explored the GeneCards database [19] (http://www.genecards.org) and DisGeNET database [20] (https://www.disgenet.org) for potential targets related to dry AMD. Although higher scores in the GeneCards database indicate a strong association between the target and the disease, there were too few relevant targets for dry AMD in several databases (1056 relevant targets in the GeneCards database and only 12 relevant targets in the DisGeNET database) and therefore no targets greater than the median were set as potential targets here. After merging the targets in the two databases, the potential targets for dry AMD can be obtained by deleting the duplicate targets.

2.3. SDSZT-Dry AMD PPI Data. To clarify the interactions between SDSZT-associated targets and dry AMD-related targets, we first used the Venny 2.1.0 (https://bioinfogp.cnb. csic.es/tools/venny/) to get intersection targets. The acquired targets are then used to construct a protein-polymer interaction (PPI) network model from the STRING 11.0 database $[21,22]$ (http://string-db.org). The biological genus is set to "Homo sapiens" and the minimum required interaction score is set to "highest confidence" $(>0.900)$; the rest are default settings to generate the PPI network diagram. Using Cytoscape 3.7.1, the network is analyzed by CytoNCA to calculate the betweeness centrality (BC), closeness centrality (CC), degree centrality (DC), and the eigenvector centrality (EC); the mean value is calculated and then filtered. The PPI network was further analyzed using the MCODE [23] plugin in Cytoscape 3.7.1.

2.4. Gene Ontology and Pathway Enrichment Analysis. The combination of functional enrichment, interactomic analysis, gene annotation, and search in Metascape is an effective tool for comprehensive analysis and interpretation of OMICs-based research in the era of big data [24]. Therefore, SDSZT-dry AMD cross-targets were imported into Metascape (http://metascape.org/gp/index.html), the $P$ value $<0.01$ was set, and enrichment analysis was performed. Using the Metascape database and the Microbiology Letter Platform (http://www.bioinformatics.com.cn/), we have conducted a number of studies on molecular function (MF), biological process (BP), and cellular component (CC). The top 20 functionally annotated catalogs and signaling pathways are selected for analysis, and the results are presented as bar and bubble plots.

2.5. SDSZT-Dry AMD-Pathway PPI Data. Cytoscape 3.7.1 was used to construct a network diagram of the constituents of SDSZT-dry AMD target-pathway network, and Cytoscape 3.7.1 built-in tools were used to analyze the network topology parameters of the active ingredients and targets, including Degree, BetweennessCentrality, and ClosenessCentrality. The network topology parameters were then 
used to determine the core targets and the main active ingredients that exerted drug effects.

\section{Results}

3.1. Candidate Compound Screening for SDSZT and Potential Target Prediction for SDSZT. Initially, we obtained 327 kinds of chemical compositions of Rehmanniae Radix Praeparata, 1042 kinds of Ligustrum lucidum, 2190 kinds of Mori Fructus, 1007 kinds of Paeonia albiflora, 871 kinds of Rhizoma Dioscoreae, 179 kinds of Alisma orientale, 39 kinds of Schisandra chinensis, 22 kinds of Radix Polygoni Multiflori Preparata, 60 kinds of Ophiopogon japonicus, and 49 kinds of Radix Rehmanniae. After ADME screening, there were 2 kinds of chemical composition in Rehmanniae Radix Praeparata, 10 kinds of Ligustrum lucidum, 6 kinds of Mori Fructus, 6 kinds of Paeonia albiflora, 15 kinds of Rhizoma Dioscoreae, 7 kinds of Alisma orientale, 5 kinds of Schisandra chinensi, 4 kinds of Radix Polygoni Multiflori Preparat, 2 kinds of Ophiopogon japonicus, and 2 kinds of Radix Rehmannia (Table 1). Finally, the chemical targets were 34 for Rehmanniae Radix Praeparata, 360 for Ligustrum lucidum, 195 for Mori Fructus, 123 for Paeonia albiflora, 144 for Rhizoma Dioscoreae, 9 for Alisma orientale, 7 for Schisandra chinensia, 49 for Radix Polygoni Multiflori Preparat, 12 for Ophiopogon japonicu, and 4 for Radix Rehmannia. After merging and deleting duplicate values, a total of 247 drug targets were collated. We then created a network diagram of the SDSZT herbs-active ingredient-drug target (Figure 1).

3.2. Collection of Known Dry AMD-Related Targets. 1056 dry AMD targets were obtained from the GeneCards database, and due to the small number of targets obtained, the score range was not set for screening. In combination with the DisGeNET database, 11 targets were obtained for nonexudative age-related macular degeneration, and 1059 dry AMD targets were obtained for dry AMD after deleting the duplicates.

3.3. SDSZT-Dry AMD PPI Data. The screened targets of SDSZT active ingredients and dry AMD disease were imported into the online Venny analysis website, and the intersection targets were plotted in the Venny plot, resulting in 107 common targets of the ingredients of SDSZT and dry AMD obtained (Figure 2). The obtained intersection targets were imported into the STRING 11.0 platform, and the PPI network of SDSZT was obtained, with 2210 interacting edges and an average node degree value of 41.3 (Figure 3(a)). In order to further clarify the potential targets and proteinprotein interactions at key nodes, the CSV files obtained in STRING 11.0 were imported into Cytoscape 3.7.1 software, and the network was analyzed by cytoNCA. The calculated values of BC, CC, DC, and EC were 0.776567196, $0.623529412,42$, and 0.091691375 , respectively. Fifty-three core targets were selected based on greater-than-mean values (Figure 3(b)). Among them, ALB, AKT1, IL6, TNF, TP53, VEGFA, CASP3, PTGS2, JUN, and MAPK1 are the top 10 targets of degree value. There are tightly connected regions in the PPI network, which may represent molecular complexes called module [25], and the MCODE plugin in Cytoscape 3.7.1 is used to mine the interacting tightly connected regions in the PPI network to obtain the modules (Figure 3(c)).

3.4. Gene Ontology and Pathway Enrichment Analysis. The signaling pathways of the targets associated with SDSZT for dry AMD were obtained from the analysis of the Metascape website. The results were then visualized by Cytoscape 3.7.1 software and the Microbiology platform. The result is a target that is inextricably linked to dry AMD.

The main biological processes involved in SDSZT include response to inorganic substance, response to toxic substance, response to lipopolysaccharide, and reactive oxygen species metabolic process, apoptotic signaling pathway, response to wounding, and response to extracellular stimulus (Figure 4(a)). The main pathways involved are pathways in cancer, fluid shear stress and atherosclerosis, and TNF signaling pathway (Figure 4(d)). The enrichment targets of target-pathway enrichment results are shown in Table 2, and the top 20 filtered pathway-target network diagrams produced by Cytoscape 3.7.1 are shown in Figure 4(e).

Relevant targets regulate the function of dry AMD, which is mainly enriched in cytokine receptor binding, transcription factor binding, protein domain specific binding, kinase binding, phosphatase binding, antioxidant activity, and oxidoreductase activity (Figure 4(b)).

3.5. SDSZT-Dry AMD-Pathway PPI Data. Cytoscape 3.7.1 analyzed the SDSZT-disease target-pathway network, as shown in Figure 5. By backpropagation, 33 compounds of SDSZT acting on dry AMD were obtained, and the network diagram included 725 nodes. The topological parameters of the SDSZT network were analyzed by NetworkAnalyzer, and the core components and core targets were obtained. The analysis showed that D1 quercetin had a degree of 162 , a betweenness of 0.4159 , and a closeness of 0.608 , predicting quercetin to be the main component of SDSZT for the treatment of dry AMD, followed by C2 kaempferol, with a degree of 54, a betweenness of 0.05453 , and a closeness of 0.4270 , and NZ6 luteolin, with a degree of 32 , a betweenness of 0.0705 , and a closeness of 0.4318 ; C1 $\beta$-sitosterol has a degree of 19 , a betweenness of 0.0075 , and a closeness of 0.38 ; SS $3 \beta$-carotene has a degree of 17 , a betweenness of 0.0259 , and a closeness of 0.3897 ; B1 stigmasterol had a degree of 14 , a betweenness of 0.0214 , and a closeness of 0.3535 (Table 3).

PTGS2, with a network degree of 29 , a betweenness of 0.1304 , and a closeness of 0.5084 in the network, was predicted to be the most important target of SDSZT for the treatment of dry AMD. Next, AKT1, PTGS1, TNF, JUN, BCL2, CASP3, PIK3CG, AR, and BAX were also relatively important regulatory targets (Table 4 ). 
TABLE 1: The main ingredients of SDSZT.

\begin{tabular}{|c|c|c|c|c|c|}
\hline Herb & Mol ID & Label & Active ingredient & OB & $\mathrm{DL}$ \\
\hline \multirow{2}{*}{$\begin{array}{l}\text { Rehmanniae Radix } \\
\text { Praeparata }\end{array}$} & MOL000359 & A1 & Sitosterol & 36.91 & 0.75 \\
\hline & MOL000449 & $\mathrm{B} 1$ & Stigmasterol & 43.83 & 0.76 \\
\hline \multirow{4}{*}{ Mori Fructus } & MOL006209 & SS1 & Cyanin & 47.42 & 0.99 \\
\hline & MOL000737 & SS2 & Morin & 46.23 & -0.77 \\
\hline & MOL002773 & SS3 & Beta-carotene & 37.18 & 1.52 \\
\hline & MOL000098 & D1 & Quercetin & 46.43 & -0.77 \\
\hline \multirow{5}{*}{ Schisandra chinensis } & MOL009199 & WWZ1 & Interiotherin $\mathrm{B}$ & 31.76 & 0.43 \\
\hline & MOL009213 & WWZ5 & Kadsulignan B & 30.63 & 0.24 \\
\hline & MOL009219 & WWZ8 & Neokadsuranic acid C & 35.4 & 0.26 \\
\hline & MOL009220 & WWZ9 & Kadsulignan & 33.35 & 0.89 \\
\hline & MOL009235 & WWZ13 & Angusifolin B & 34.82 & 0.42 \\
\hline \multirow{4}{*}{$\begin{array}{l}\text { Radix Polygoni } \\
\text { Multiflori Praeparata }\end{array}$} & MOL001771 & SW1 & Poriferast-5-en-3beta-ol & 36.91 & 0.75 \\
\hline & MOL002268 & SW2 & Rhein & 47.07 & 0.28 \\
\hline & MOL004373 & SW3 & Anhydroicaritin & 45.41 & 0.44 \\
\hline & MOL000359 & A1 & Sitosterol & 36.91 & 0.75 \\
\hline \multirow{8}{*}{ Paeonia albiflora } & MOL000359 & A1 & Sitosterol & 36.91 & 0.75 \\
\hline & MOL000358 & $\mathrm{C} 1$ & Beta-sitosterol & 36.91 & 0.75 \\
\hline & MOL000422 & $\mathrm{C} 2$ & Kaempferol & 41.88 & 0.24 \\
\hline & MOL001918 & BS1 & Paeoniflorgenone & 87.59 & 0.37 \\
\hline & MOL001919 & $\mathrm{BS} 2$ & $\begin{array}{c}\text { (3S,5 R,8R,9 R,10S,14S)-3,17-dihydroxy-4,4,8,10,14-pentamethyl- } \\
\text { 2,3,5,6,7,9-hexahydro-1h-cyclopenta[a]p }\end{array}$ & 43.56 & 0.53 \\
\hline & MOL001924 & BS3 & Paeoniflorin & 53.87 & 0.79 \\
\hline & MOL000211 & BS4 & Mairin & 55.38 & 0.78 \\
\hline & MOL000492 & BS5 & $(+)$-catechin & 54.83 & 0.24 \\
\hline \multirow{12}{*}{ Rhizoma Dioscoreae } & MOL000449 & B1 & Stigmasterol & 43.83 & 0.76 \\
\hline & MOL001559 & SY1 & Piperlonguminine & 30.71 & 0.18 \\
\hline & MOL001736 & SY2 & $(-)$-Taxifolin & 60.51 & 0.27 \\
\hline & MOL000322 & SY3 & Kadsurenone & 54.72 & 0.38 \\
\hline & MOL005430 & SY4 & Hancinone C & 59.05 & 0.39 \\
\hline & MOL005435 & SY5 & 24-Methylcholest-5-enyl-3belta-O-glucopyranoside_qt & 37.58 & 0.72 \\
\hline & MOL005438 & SY6 & Campesterol & 37.58 & 0.7 \\
\hline & MOL005440 & SY7 & Isofucosterol & 43.78 & 0.76 \\
\hline & MOL005458 & SY8 & Dioscoreside C_qt & 36.38 & 0.87 \\
\hline & MOL000546 & SY9 & Diosgenin & 80.88 & 0.81 \\
\hline & MOL005465 & SY10 & AIDS180907 & 45.33 & 0.77 \\
\hline & MOL000953 & SY11 & CLR & 37.87 & 0.68 \\
\hline \multirow{7}{*}{ Alisma orientale } & MOL000359 & A1 & Sitosterol & 36.91 & 0.75 \\
\hline & MOL000831 & ZX1 & Alisol B monoacetate & 35.58 & 0.81 \\
\hline & MOL000849 & $\mathrm{ZX} 2$ & $16 \beta$-methoxyalisol B monoacetate & 32.43 & 0.77 \\
\hline & MOL000853 & $\mathrm{ZX} 3$ & Alisol B & 36.76 & 0.82 \\
\hline & MOL000856 & $\mathrm{ZX} 4$ & Alisol C monoacetate & 33.06 & 0.83 \\
\hline & MOL002464 & ZX5 & 1-Monolinolein & 37.18 & 0.3 \\
\hline & MOL000862 & ZX6 & $\begin{array}{c}\text { [(1S,3 R)-1-[(2R)-3,3-dimethyloxiran-2-yl]-3-[(5R,8S,9S,10S,11S,14 R)- } \\
\text { 11-hydroxy-4,4,8,10,14-pentamethyl-3-oxo-1,2,5,6,7,9,11,12,15,16- } \\
\text { decahydrocyclopenta[a]phenanthren-17-yl]butyl] acetate }\end{array}$ & 35.58 & 0.81 \\
\hline \multirow{9}{*}{ Ligustrum lucidum } & MOL000358 & $\mathrm{C} 1$ & Beta-sitosterol & 36.91 & 0.75 \\
\hline & MOL000422 & $\mathrm{C} 2$ & Kaempferol & 41.88 & 0.24 \\
\hline & MOL000098 & D1 & Quercetin & 46.43 & -0.77 \\
\hline & MOL004576 & NZ1 & Taxifolin & 57.84 & 0.27 \\
\hline & MOL005146 & NZ2 & Lucidumoside D & 48.87 & 0.71 \\
\hline & MOL005147 & NZ3 & Lucidumoside D_qt & 54.41 & 0.47 \\
\hline & MOL005190 & NZ4 & Eriodictyol & 71.79 & 0.24 \\
\hline & MOL005212 & NZ5 & Olitoriside_qt & 103.23 & 0.78 \\
\hline & MOL000006 & NZ6 & Luteolin & 36.16 & 0.25 \\
\hline \multirow{2}{*}{ Ophiopogon japonicus } & MOL001659 & MD1 & Poriferasterol & 43.83 & 0.76 \\
\hline & MOL000631 & MD2 & Coumaroyltyramine & 112.9 & 0.2 \\
\hline \multirow{2}{*}{ Radix Rehmanniae } & MOL002813 & DH1 & Aucubin & 35.56 & 0.33 \\
\hline & MOL012254 & $\mathrm{DH} 2$ & Campesterol & 37.58 & 0.71 \\
\hline
\end{tabular}




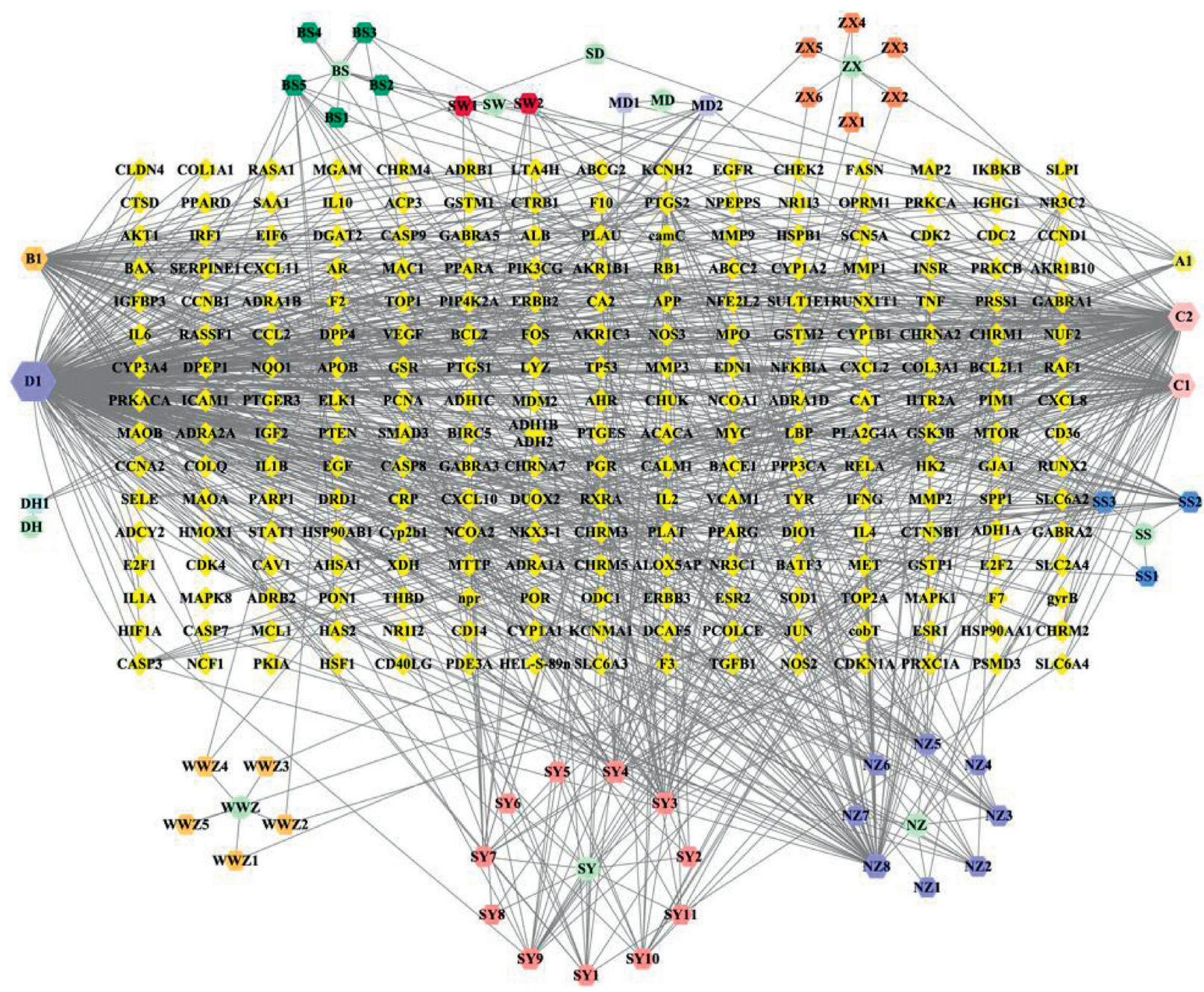

FIGURE 1: Construction of the SDSZT compound-potential target network. The compound-potential target network was constructed by linking the candidate compounds and their potential targets of the 10 herbs, which are constituents of SDSZT. Squares are for traditional Chinese medicine; hexagons are compound components; abbreviations for compounds can be found in Table 1; diamonds are targets.

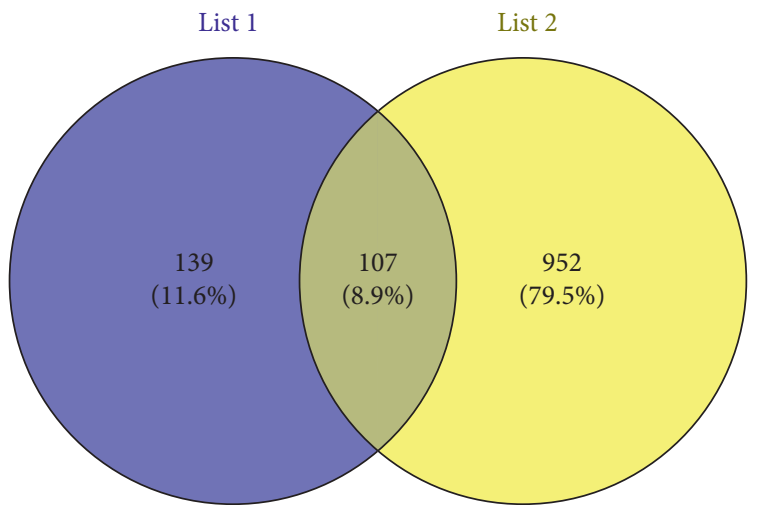

FIgURE 2: SDSZT component targets (list 1); dry AMD targets (list 2); Venny chart.

\section{Discussion}

Age-related macular degeneration is an age-related disease that is currently recognized as a high-risk blinding eye disease, and it is essential to establish an effective treatment measure in an increasingly aging society. Dry AMD is known to be a complex disease with multiple risk factors and molecular mechanisms. In the study of in vivo and in vitro experimental models of $\mathrm{AMD}$, these molecular mechanisms involving AMD can be broadly categorized as oxidative stress-mediated, antioxidant dysregulation, inflammation, dysregulation of lipid metabolism, and angiogenic dysregulation [1, 3, 26]. For dry age-related macular degeneration (AMD) and advanced geographic atrophy (GA), the treatment options available only slow the progression of the disease. However, there is currently no therapy that can restore degenerated retinal pigment epithelial (RPE) and/or photoreceptor cells. Currently, studies have focused on antibody, gene, and stem cell therapies for the treatment of dry AMD [27, 28], which are potentially effective therapies but have not yet been applied in the clinical setting in the absence of long-term validated safety profiles.

Shuang Di Shou Zhen Tablets (formerly known as Ziyin Bushen Tablets) have been used in the treatment of dry AMD for 20 years. In previous clinical studies, patients with dry AMD 


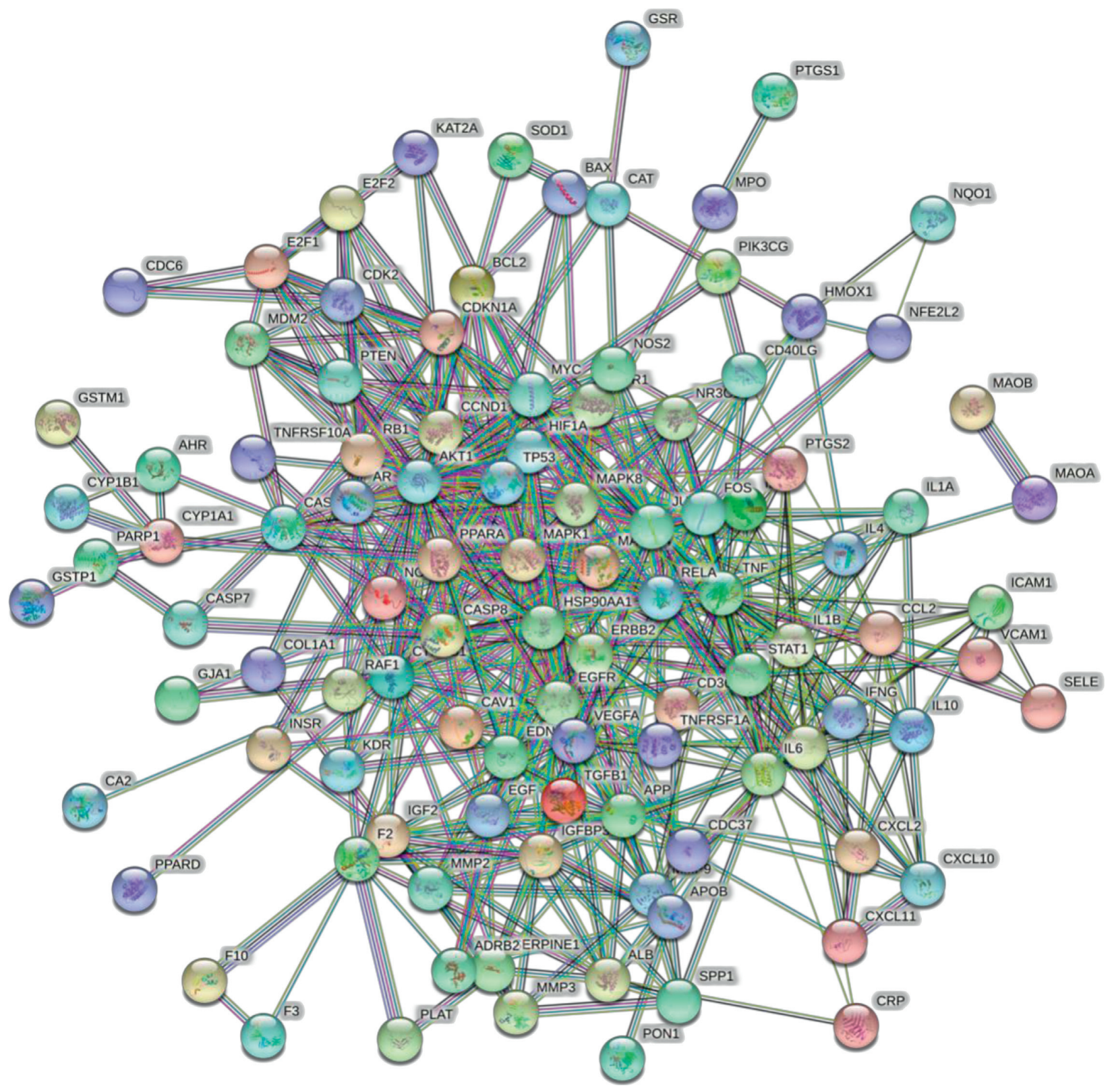

(a)

Figure 3: Continued. 


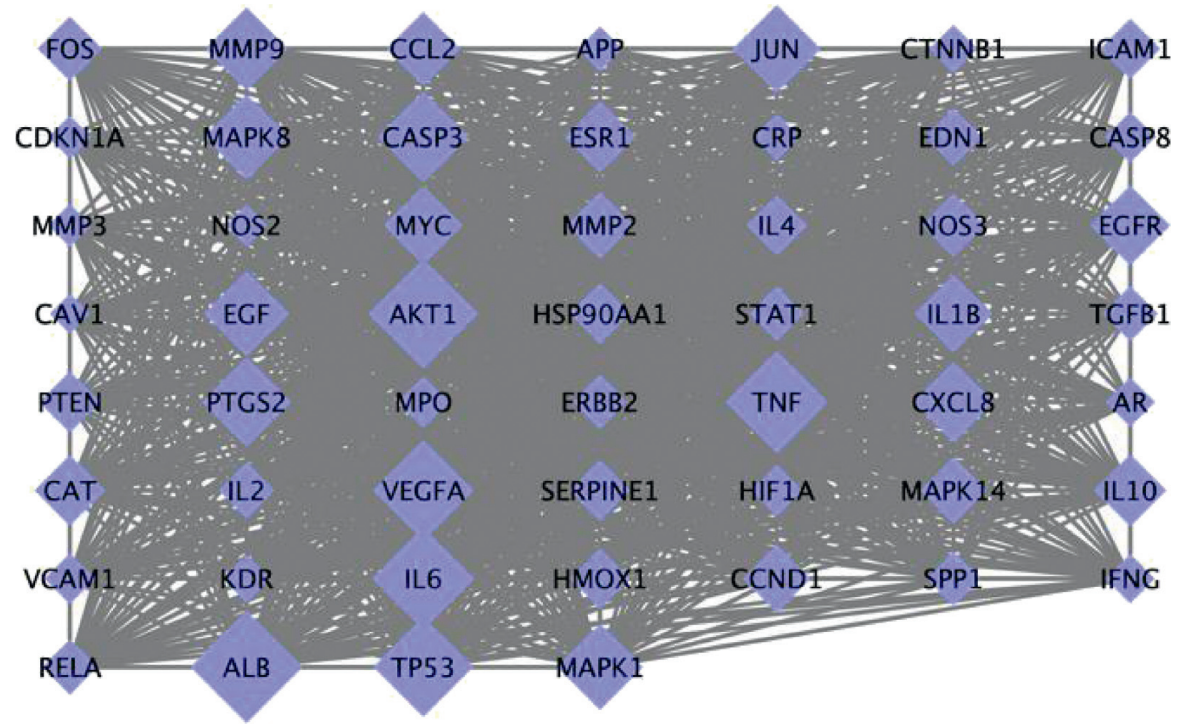

(b)
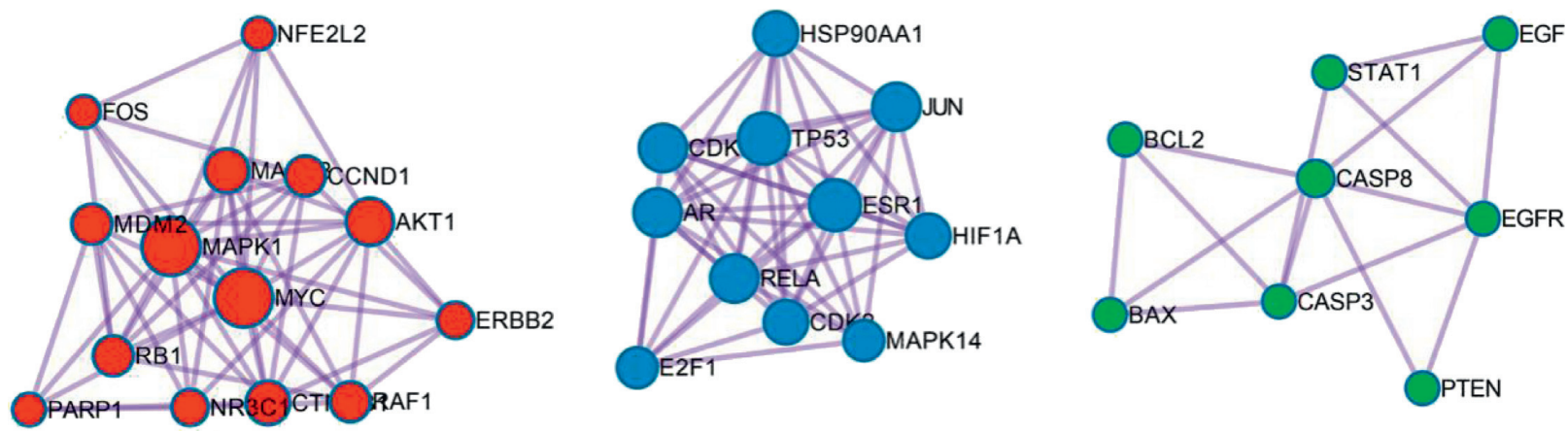

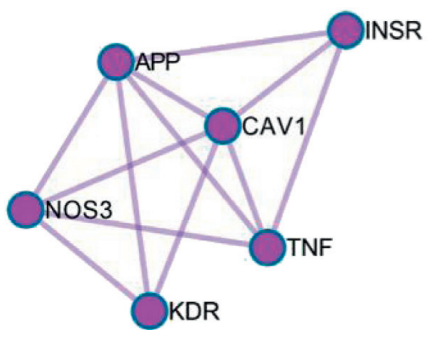

MCODE1

MCODE2

MCODE3

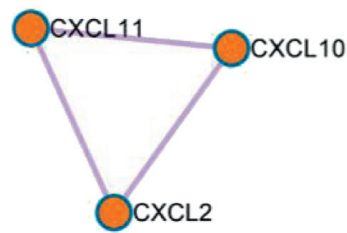

MCODE4

MCODE5

(c)

FIGURE 3: Protein-protein interaction networks. The mapping of PPI network was generated by the STRING server. (a) SDSZT-dry AMD targets PPI network (String): 107 main target genes regulated by SDSZT formula in the treatment of dry AMD and its complications. This network contains 107 nodes and 2210 edges. (b) PPI network of SDSZT-dry AMD key target. As shown in (b), the larger area of the rhombus could be considered as more important in this network. The degree value of each node in Figure 2(c) is not presented in article. (c) Module in a PPI network for SDSZT-dry AMD targets.

were found to have improved visual acuity after receiving a combination of SDSZT and supplements such as vitamin E and vitamin $\mathrm{C}$ compared to before treatment. As the number of deformation grid in Amsler table was reduced, the range of central vision defect was reduced, and there were also statistical differences on these respects between treatment group and control group (only treated with vitamin $\mathrm{E}$ and vitamin $\mathrm{C}$ ) $(P<0.05)$ [6,7], but the pharmacological mechanism of action has not been clearly understood. In this study, the active components of SDSZT for the treatment of dry AMD were 


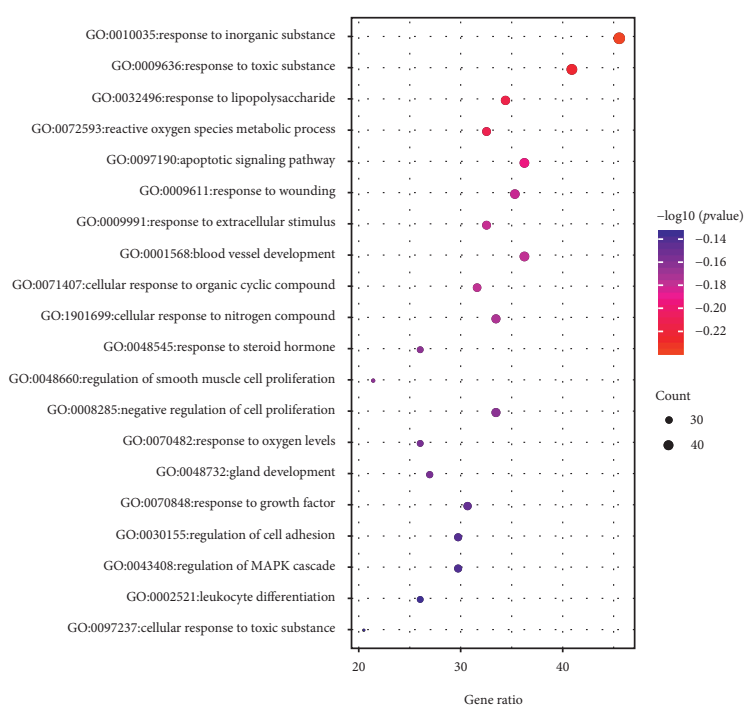

(a)

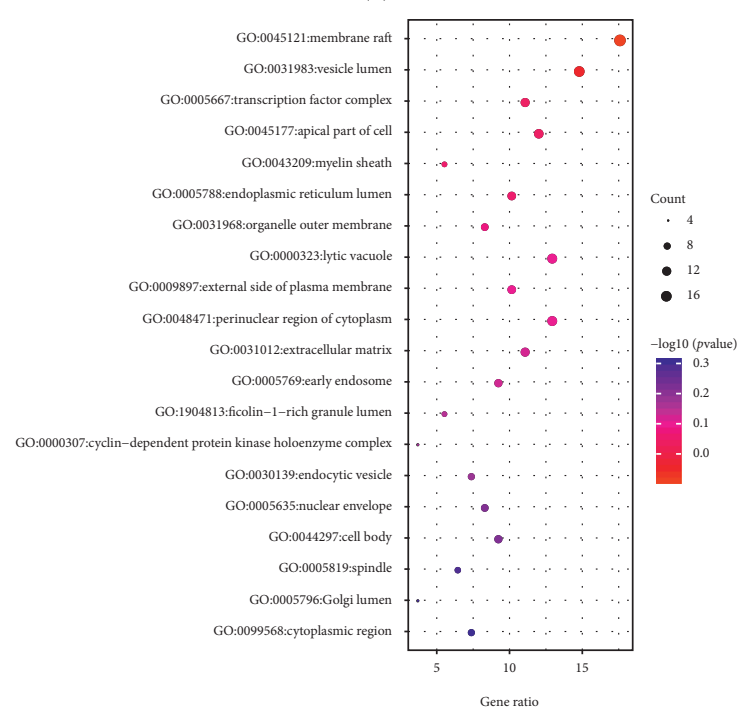

(c)

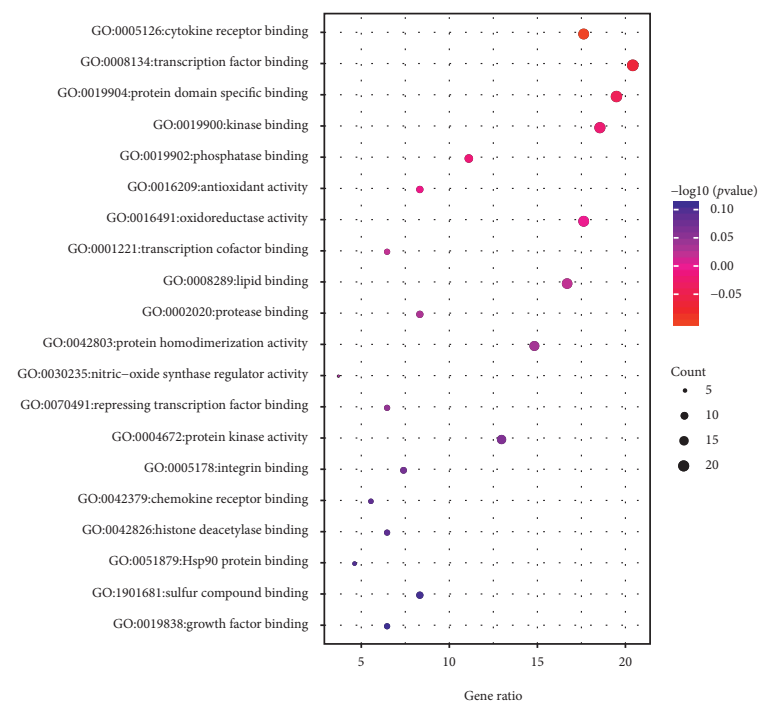

(b)

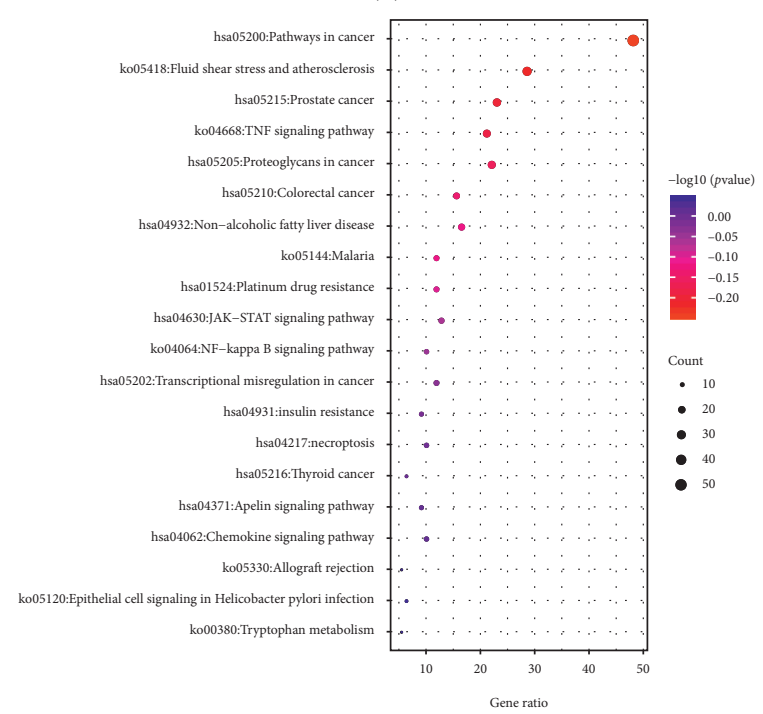

(d)

Figure 4: Continued. 


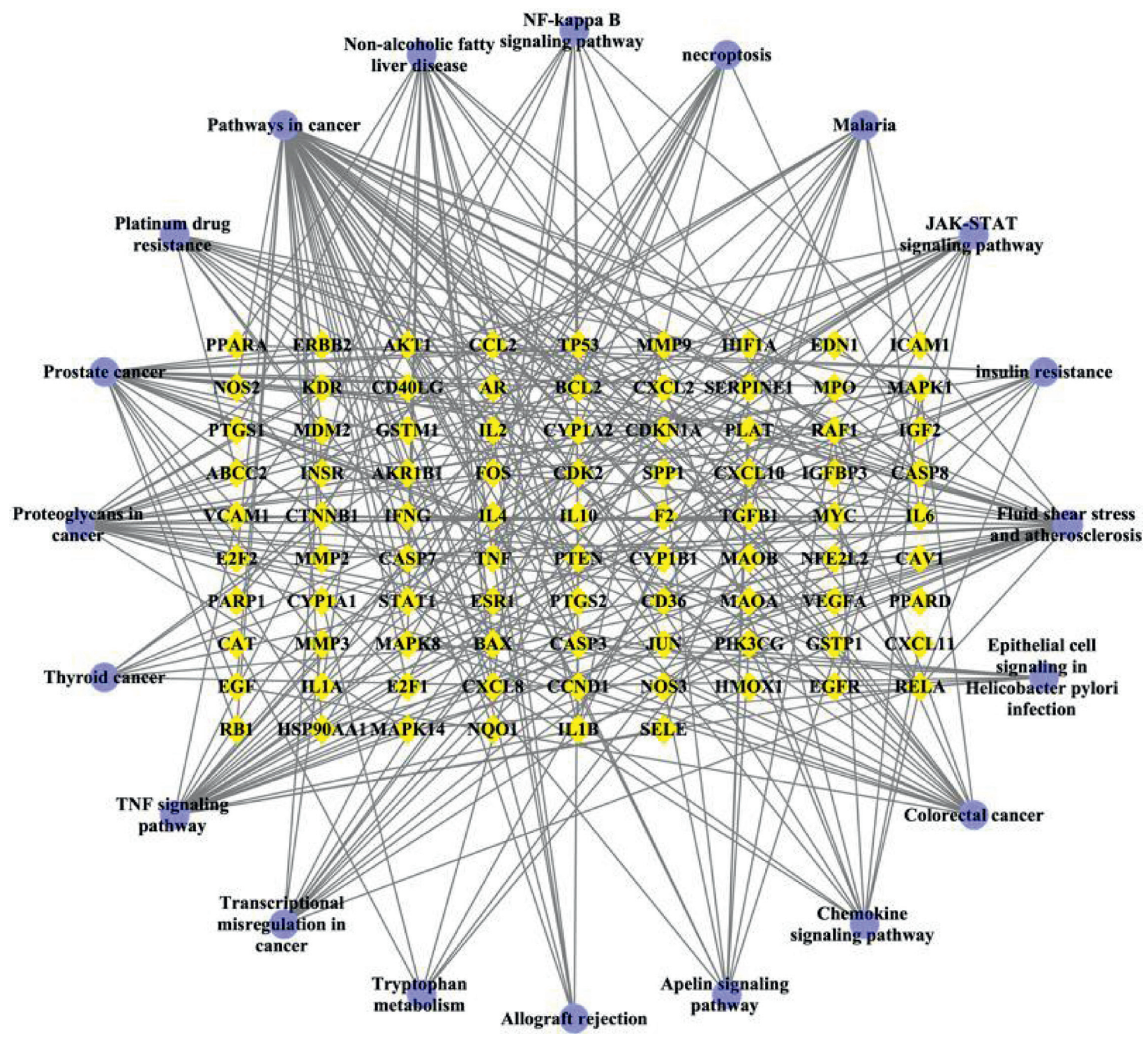

(e)

FIGURE 4: Enrichment analysis of potential targets for the main components of SDSZT. (a): GO-BP analysis; (b): GO-MF analysis; (c): GOCC analysis; (d): KEGG analysis. (e): Intersection of the top 20 KEGG pathways and targets after screening, with hexagonal pathways and rhombuses as common targets.

quercetin, kaempferol, luteolin, $\beta$-sitosterol, $\beta$-carotene, stigmasterol, etc., based on the preliminary screening by network pharmacology method. Studies on the therapeutic mechanisms of dry AMD have shown that polyphenols such as quercetin can directly scavenge reactive oxygen species (ROS) [29] and it protects ARPE-19 cells against oxidative stress induced by 4hydroxynonene (HNE), an end product of lipid peroxidation [30]. Quercetin protects RPE cells by decreasing mRNA expression of the proinflammatory IL- 6 and IL-8 and monocyte chemotactic protein 1 (MCP-1) [31] and activating the Keap1/ Nrf2/ARE pathway [32]. In vivo experiments have shown that kaempferol reduces the apoptosis of rat retinal pigment epithelial cells (RPE cells) caused by $\mathrm{NaIO} 3$ by enhancing the expression of vascular endothelial growth factor (VEGF) protein [33]. Luteolin attenuates the IL- $1 \beta$-induced increase in interleukin 6 (IL-6), interleukin 8 (IL-8), soluble intercellular adhesion molecule 1 (sICAM-1), and monocyte chemotactic protein 1 (MCP-1) production by inhibiting nuclear transcription factor-kB $(\mathrm{NF} \kappa-\mathrm{B})$ and MAPK signaling pathways to further protect ARPE19 cells [34]. Therefore, SDSZT may be used to regulate dry age-related macular degeneration through quercetin, kaempferol, and luteolin.

Based on the results of this study, the highest degree value target of SDSZT for dry age-related macular degeneration is PTSG2, while AKT1, PTGS1, TNF, JUN, BCL2, CASP3, PIK3CG, AR, and BAX are also likely to be key therapeutic targets for SDSZT. Gene Ontology analysis and KEGG pathway enrichment analysis showed that these key targets involve multiple pathways, including prostate cancer [35], TNF signaling pathway [36], NF-Kappa B signaling pathway [37], and 
TABLE 2: Enrichment analysis of candidate targets for SDSZT against dry AMD.

\begin{tabular}{|c|c|c|c|c|}
\hline $\mathrm{GO}$ & Description & Count & $\log 10(\mathrm{P})$ & Hits \\
\hline hsa05200 & Pathways in cancer & 52 & -58.12 & $\begin{array}{c}\text { AKT1|AKR1B1|AR|BAX|CCND1|BCL2|CASP3|CASP7|CASP8| } \\
\text { CDK2|CDKN1A|CTNNB1|NQO1|E2F1|E2F2|EDN1|EGF|EGFR| } \\
\text { ERBB2|ESR1|F2|FOS|GSTM1|GSTP1|HIF1A|HMOX1|HSP90AA1| } \\
\text { IFNG|IGF2|IL2|IL4|IL6|CXCL8|JUN|MDM2|MMP2|MMP9|MYC| } \\
\text { NFE2L2|NOS2|PPARD|MAPK1|MAPK8|PTEN|PTGS2|RAF1|RB1| } \\
\text { RELA|STAT1|TGFB1|TP53|VEGFA }\end{array}$ \\
\hline ko05418 & $\begin{array}{l}\text { Fluid shear stress and } \\
\text { atherosclerosis }\end{array}$ & 31 & -45.9 & $\begin{array}{c}\text { AKT1|BCL2|CAV1|MAPK14|CTNNB1|NQO1|EDN1|FOS|GSTM1| } \\
\text { GSTP1|HMOX1|HSP90AA1|ICAM1|IFNG|IL1A|IL1B|JUN|KDR| } \\
\text { MMP2|MMP9|NFE2L2|NOS3|PLAT|MAPK8|RELA|CCL2|SELE| } \\
\text { TNF|TP53|VCAM1|VEGFA }\end{array}$ \\
\hline hsa05215 & Prostate cancer & 25 & -38.22 & $\begin{array}{l}\text { AKT1|AKR1B1|AR|CCND1|BCL2|CDK2|CDKN1A|CTNNB1| } \\
\text { E2F1|E2F2|EGF|EGFR|ERBB2|GSTP1|HSP90AA1|MDM2|MMP3| } \\
\text { MMP9|PLAT|MAPK1|PTEN|RAF1|RB1|RELA|TP53 }\end{array}$ \\
\hline ko04668 & TNF signaling pathway & 23 & -33.56 & $\begin{array}{c}\text { AKT1|CASP3|CASP7|CASP8|MAPK14|EDN1|FOS|CXCL2|ICAM1| } \\
\text { IL1B|IL6|CXCL10|JUN|MMP3|MMP9|MAPK1|MAPK8|PTGS2| } \\
\text { RELA|CCL2|SELE|TNF|VCAM1 }\end{array}$ \\
\hline hsa05205 & Proteoglycans in cancer & 24 & -27.71 & $\begin{array}{c}\text { AKT1|CCND1|CASP3|CAV1|CDKN1A|MAPK14|CTNNB1|EGFR| } \\
\text { ERBB2|ESR1|HIF1A|IGF2|IL6|KDR|MDM2|MMP2|MMP9|MYC| } \\
\text { MAPK1|RAF1|TGFB1|TNF|TP53|VEGFA }\end{array}$ \\
\hline hsa05210 & Colorectal cancer & 17 & -23.46 & $\begin{array}{c}\text { AKT1|BAX|CCND1|BCL2|CASP3|CDKN1A|CTNNB1|EGF|EGFR| } \\
\text { FOS|JUN|MYC|MAPK1|MAPK8|RAF1|TGFB1|TP53 }\end{array}$ \\
\hline hsa04932 & Nonalcoholic fatty liver disease & 18 & -20.48 & $\begin{array}{c}\text { AKT1|BAX|CASP3|CASP7|CASP8|IL1A|IL1B|IL6|CXCL8|INSR|JUN| } \\
\text { PPARA|MAPK8|PTGS1|PTGS2|RELA|TGFB1|TNF }\end{array}$ \\
\hline ko05144 & Malaria & 13 & -20.39 & $\begin{array}{l}\text { CD36|CD40LG|ICAM1|IFNG|IL1B|IL6|CXCL8|IL10|CCL2|SELE| } \\
\text { TGFB1|TNF|VCAM1 }\end{array}$ \\
\hline hsa01524 & Platinum drug resistance & 13 & -17.9 & $\begin{array}{c}\text { AKT1|BAX|BCL2|CASP3|CASP8|CDKN1A|ABCC2|ERBB2|GSTM1| } \\
\text { GSTP1|MDM2|MAPK1|TP53 }\end{array}$ \\
\hline hsa04630 & JAK-STAT signaling pathway & 14 & -14.36 & $\begin{array}{l}\text { AKT1|CCND1|BCL2|CDKN1A|EGF|EGFR|IFNG|IL2|IL4|IL6|IL10| } \\
\text { MYC|RAF1|STAT1 }\end{array}$ \\
\hline ko04064 & NF-kappa B signaling pathway & 11 & -13.05 & $\begin{array}{l}\text { PARP1|BCL2|CD40LG|CXCL2|ICAM1|IL1B|CXCL8|PTGS2|RELA| } \\
\text { TNF|VCAM1 }\end{array}$ \\
\hline hsa05202 & $\begin{array}{l}\text { Transcriptional misregulation in } \\
\text { cancer }\end{array}$ & 13 & -11.96 & $\begin{array}{l}\text { BAX|CDKN1A|IGFBP3|IL6|CXCL8|MDM2|MMP3|MMP9|MPO| } \\
\text { MYC|PLAT|RELA|TP53 }\end{array}$ \\
\hline hsa04931 & Insulin resistance & 10 & -10.71 & AKT1|CD36|IL6|INSR|NOS3|PPARA|MAPK8|PTEN|RELA|TNF \\
\hline hsa04217 & Necroptosis & 11 & -10.31 & $\begin{array}{l}\text { PARP1|BAX|BCL2|CASP8|HSP90AA1|IFNG|IL1A|IL1B|MAPK8| } \\
\text { STAT1|TNF }\end{array}$ \\
\hline hsa05216 & Thyroid cancer & 7 & -9.87 & BAX|CCND1|CDKN1A|CTNNB1|MYC|MAPK1|TP53 \\
\hline hsa04371 & Apelin signaling pathway & 10 & -9.6 & $\begin{array}{c}\text { AKT1|CCND1|NOS2|NOS3|SERPINE1|PIK3CG|PLAT|MAPK1| } \\
\text { RAF1|SPP1 }\end{array}$ \\
\hline hsa04062 & Chemokine signaling pathway & 11 & -9.45 & $\begin{array}{l}\text { AKT1|CXCL2|CXCL8|CXCL10|PIK3CG|MAPK1|RAF1|RELA|CCL2| } \\
\text { CXCL11|STAT1 }\end{array}$ \\
\hline ko05330 & Allograft rejection & 6 & -8.1 & CD40LG|IFNG|IL2|IL4|IL10|TNF \\
\hline ko05120 & $\begin{array}{l}\text { Epithelial cell signaling in } \\
\text { Helicobacter pylori infection }\end{array}$ & 7 & -8.11 & CASP3|MAPK14|EGFR|CXCL8|JUN|MAPK8|RELA \\
\hline ko00380 & Tryptophan metabolism & 6 & -8.03 & CAT|CYP1A1|CYP1A2|CYP1B1|MAOA|MAOB \\
\hline
\end{tabular}




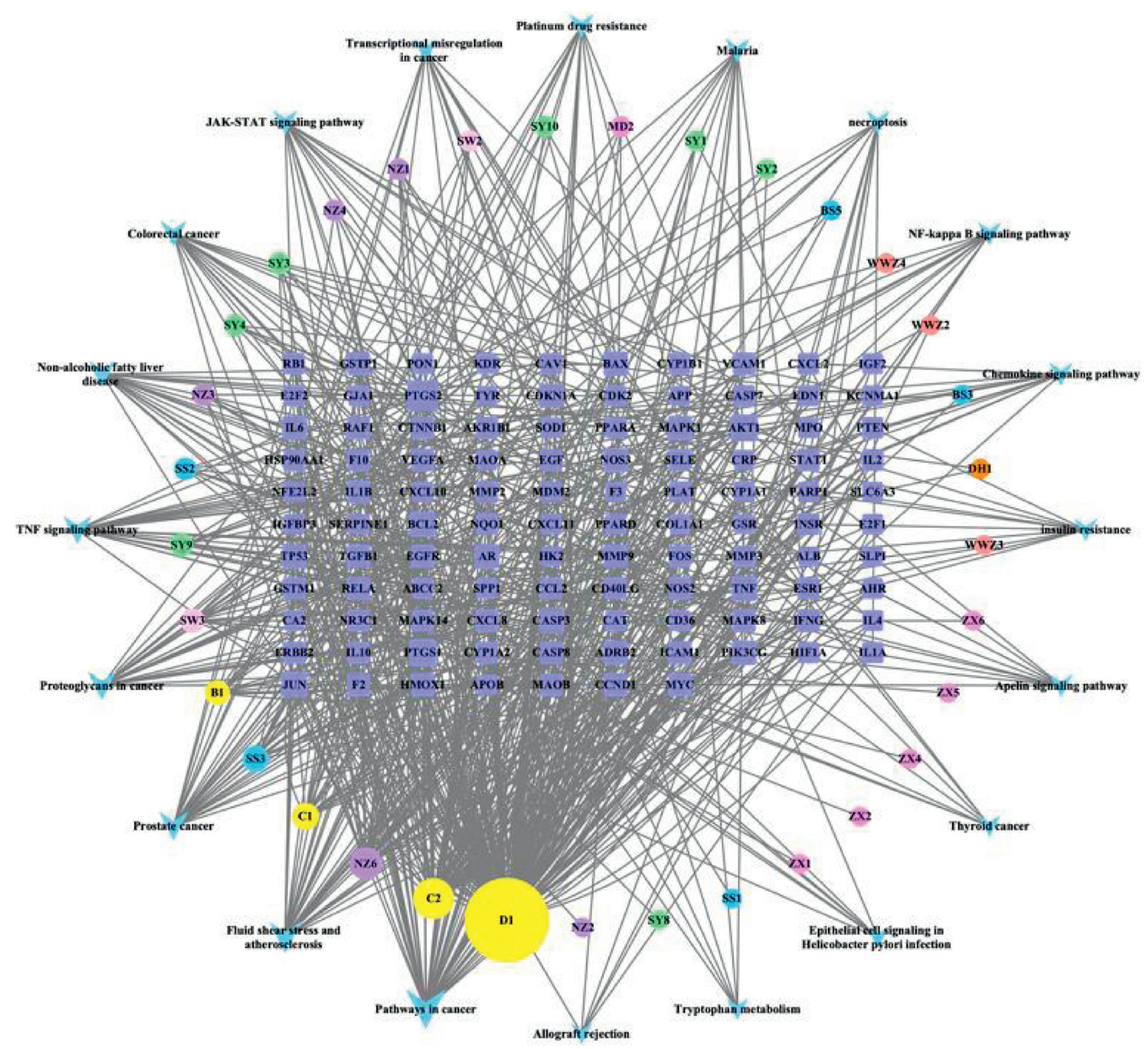

FIGURE 5: SDSZT component-dry AMD target-pathway network diagram. Triangles are pathways; circles are active components of drugs; different colors are components of different drugs; squares are targets shared by disease drugs. Refer to Table 1 for Chinese herbal abbreviations.

JAK-STAT signaling pathway [38], all of which are closely related to the AMD development process. PTGS2, also known as cyclooxygenase (COX2), has been reported to be involved in the AMD process as a key enzyme in the inflammatory response, and is highly expressed in retinal pigment epithelial cells [39]. JUN, also known as transcription factor ap-1, can be activated by 4-hydroxyhexenal- (HHE-) under the control of cyanidin-3-glucoside $(\mathrm{C} 3 \mathrm{G})$ and is associated with AMD inflammation [40]. The expression of phosphatidylinositol 3 kinase (PIK3CG) was upregulated when bevacizumab treated RPE cells in an AMD model [41], suggesting that PIK3CG may be a target for the treatment of AMD. Current information on the modes of RPE death in AMD disease includes apoptosis, necrosis, autophagy, and ferroptosis [42]. The downregulation of the apoptosis suppressor gene $B c l-2$ and the upregulation of the proapoptosis gene $B a x$ have been shown to be the main causes of apoptosis in RPE cells in the AMD model [43].

The results of this study indicate that a single monomer compound of SDSZT can regulate different targets, and a single target can interfere with different biological processes and signaling pathways. This embodies the function characteristics of the multipathway, multitarget function of SDSZT. At the same time, it is clear that the whole network can be modulated by modulating a single monomer or an important target or multiple targets in the pharmacological network. This provides a scientific basis for the clinical use of SDSZT for dry age-related macular degeneration and a new direction for exploring the potential mechanisms of SDSZT for dry age-related macular degeneration. However, network pharmacology methods have certain limitations, and validation of the predicted targets and monomer compounds is 
TABLE 3: SDSZT main active ingredient network node characteristic. The chemical structure diagram is from the TCMSP Database.

\begin{tabular}{|c|c|c|c|c|c|}
\hline MOLID & Molecule name & Degree & Betweenness & Closeness & Chemical structure \\
\hline MOL000098 & Quercetin & 162 & 0.4159 & 0.608 & \\
\hline MOL000422 & Kaempferol & 54 & 0.05453 & 0.4270 & \\
\hline MOL000006 & Luteolin & 32 & 0.0705 & 0.4318 & \\
\hline MOL000358 & Beta-sitosterol & 19 & 0.0075 & 0.38 & \\
\hline MOL002773 & Beta-carotene & 17 & 0.0259 & 0.3897 & \\
\hline MOL000449 & Stigmasterol & 14 & 0.0214 & 0.3535 & \\
\hline
\end{tabular}


TABLE 4: SDSZT main active component target network node characterization parameters.

\begin{tabular}{lccc}
\hline Target & Degree & Betweenness & Closeness \\
\hline PTGS2 & 29 & 0.13042 & 0.50836 \\
AKT1 & 19 & 0.02642 & 0.46200 \\
PTGS1 & 19 & 0.05248 & 0.47059 \\
TNF & 15 & 0.02098 & 0.44058 \\
JUN & 15 & 0.01317 & 0.44060 \\
BCL2 & 15 & 0.01198 & 0.43804 \\
CASP3 & 15 & 0.01202 & 0.44315 \\
PIK3CG & 13 & 0.01881 & 0.43304 \\
AR & 13 & 0.03148 & 0.44838 \\
BAX & 13 & 0.00913 & 0.42577 \\
\hline
\end{tabular}

necessary as well to further demonstrate the clinical therapeutic potential of SDSZT and then clarify the exact regulation target of SDSZT.

\section{Data Availability}

The data supporting this network pharmacology analysis are from previously reported studies and databases, which have been cited. The processed data are available from the corresponding author upon request.

\section{Conflicts of Interest}

The authors declare that they have no conflicts of interest.

\section{Authors' Contributions}

Yue Fang and Xinquan Liu contributed equally to this work. Yue Fang, Xinquan Liu, and Jing Su conceived and designed the study and wrote the manuscript. Yue Fang and Xinquan Liu were responsible for data collation and extraction and performed the data analysis. Xinquan Liu and Jing $\mathrm{Su}$ performed supervision and project administration. All authors read and approved the final manuscript.

\section{Acknowledgments}

The authors acknowledge the web database platform and software for data analysis. This study was totally supported by the National Natural Science Foundation of China (Grant No. 81904257).

\section{References}

[1] S. Abokyi, C.-H. To, T. T. Lam, and D. Y. Tse, "Central role of oxidative stress in age-related macular degeneration: evidence from a review of the molecular mechanisms and animal models," Oxidative Medicine and Cellular Longevity, vol. 2020, Article ID 7901270, 19 pages, 2020.

[2] E. Kassa, T. A. Ciulla, R. M. Hussain, and P. U. Dugel, "Complement inhibition as a therapeutic strategy in retinal disorders," Expert Opinion on Biological Therapy, vol. 19, no. 4, pp. 335-342, 2019.

[3] J. T. Handa, C. D. Rickman, A. D. Dick et al., "A systems biology approach towards understanding and treating nonneovascular age-related macular degeneration," Nature Communication, vol. 10, no. 1, p. 3347, 2019.
[4] G. Li, Y. Shao, and J. Yin, "Early age-related macular degeneration treated with emayaoling acupuncture technique: a randomized controlled trial," Zhongguo Zhen Jiu, vol. 37, no. 12, pp. 1294-1298, 2017.

[5] H. Bing-lin et al., "Clinical observation of siwu wuzi pill in the treatment of dry age-related macular degeneration of liver and kidney deficiency type," Journal of Jiang Xi University of TCM, vol. 33, no. 1, pp. 39-41, 2021.

[6] S. Jing and L. Xinquan, "Survey of Ziyin Bushen tablets on vision-related quality of life in patients with dry age-related macular degeneration China," Journal of Chinese Ophthalmology, vol. 27, no. 4, pp. 223-117, 2017.

[7] S. Jing, L. Xinquan, and Z. Yinjian, "Effects observation of Yin-nourishing and kidney-tonifying pills on age-related macular degeneration," Hebei Journal of Traditional Chinese Medicine, vol. 39, no. 6, pp. 835-862, 2017.

[8] P.-C. Liao, M.-H. Lai, K.-P. Hsu et al., "Identification of $\beta$-sitosterol as in vitro anti-inflammatory constituent in moringa oleifera," Journal of Agricultural and Food Chemistry, vol. 66, no. 41, pp. 10748-10759, 2018.

[9] Y. Yin, X. Liu, J. Liu et al., "Beta-sitosterol and its derivatives repress lipopolysaccharide/d-galactosamine-induced acute hepatic injury by inhibiting the oxidation and inflammation in mice," Bioorganic and Medicinal Chemistry Letters, vol. 28, no. 9, pp. 1525-1533, 2018.

[10] Y.-S. Kim, X.-F. Li, K.-H. Kang, B. Ryu, and S. K. Kim, "Stigmasterol isolated from marine microalgae Navicula incerta induces apoptosis in human hepatoma HepG2 cells," BMB Reports, vol. 47, no. 8, pp. 433-438, 2014.

[11] F. M. Michelini, M. G. Lombardi, C. A. Bueno, A. Berra, M. E. Sales, and L. E. Alché, "Synthetic stigmasterol derivatives inhibit capillary tube formation, herpetic corneal neovascularization and tumor induced angiogenesis," Steroids, vol. 115, pp. 160-168, 2016.

[12] A. O. Antwi, D. D. Obiri, N. Osafo, L. B. Essel, A. D. Forkuo, and C. Atobiga, "Stigmasterol alleviates cutaneous allergic responses in rodents," Biomed Research International, vol. 2018, Article ID 3984068, 13 pages, 2018.

[13] J. Ru, P. Li, J. Wang et al., "TCMSP: a database of systems pharmacology for drug discovery from herbal medicines," Journal of Cheminformatics, vol. 6, p. 13, 2014.

[14] L. Huang, D. Xie, Y. Yu et al., "TCMID 2.0: a comprehensive resource for TCM," Nucleic Acids Research, vol. 46, no. D1, pp. D1117-D1120, 2018.

[15] X. Xu, W. Zhang, C. Huang et al., "A novel chemometric method for the prediction of human oral bioavailability," International Journal of Molecular Sciences, vol. 13, no. 6, pp. 6964-6982, 2012.

[16] S. Tian, J. Wang, Y. Li, X. Xu, and T. Hou, "Drug-likeness analysis of traditional Chinese medicines: prediction of drug- 
likeness using machine learning approaches," Molecular Pharmaceutics, vol. 9, no. 10, pp. 2875-2886, 2012.

[17] S. Wang1, H. Wang3, and Y. Lu, "Tianfoshen oral liquid: a CFDA approved clinical traditional Chinese medicine, normalizes major cellular pathways disordered during colorectal carcinogenesis," Oncotarget, vol. 8, no. 9, pp. 14549-14569, 2017.

[18] B. Demchak, T. Hui, M. Reich et al., "Cytoscape: the network visualization tool for GenomeSpace workflows," F1000 Research, vol. 3, p. 151, 2014.

[19] S. Fishilevich et al., "Genic insights from integrated human proteomics in genecards," Database (Oxford), vol. 2016, 2016.

[20] J. Piñero, À. Bravo, N. Queralt-Rosinach et al., "DisGeNET: a comprehensive platform integrating information on human disease-associated genes and variants," Nucleic Acids Research, vol. 45, no. D1, pp. D833-D839, 2017.

[21] D. Szklarczyk, A. L. Gable, D. Lyon et al., "STRING v11: protein-protein association networks with increased coverage, supporting functional discovery in genome-wide experimental datasets," Nucleic Acids Research, vol. 47, no. D1, pp. D607-D613, 2019.

[22] D. Szklarczyk, A. Franceschini, S. Wyder et al., "STRING v10: protein-protein interaction networks, integrated over the tree of life," Nucleic Acids Research, vol. 43, no. D1, pp. D447-D452, 2015.

[23] T. Li, S. Gao, L. Hui, J. Yu, and H. Li, "Identification of hub genes with prognostic values in gastric cancer by bioinformatics analysis," World Journal of Surgical Oncology, vol. 16, no. 1, p. 114, 2018.

[24] Y. Zhou, B. Zhao, L. Pache et al., "Metascape provides a biologist-oriented resource for the analysis of systems-level datasets," Nature Communication, vol. 10, no. 1, p. 1523, 2019.

[25] D. Vella, S. Marini, F. Vitali, and D. D. Silvestre, "MTGO: PPI network analysis via topological and functional module identification," Scientific Reports, vol. 8, no. 1, p. 5499, 2018.

[26] J. Blasiak, "Senescence in the pathogenesis of age-related macular degeneration," Cellular and Molecular Life Sciences, vol. 77, no. 5, pp. 789-805, 2020.

[27] E. Akyol and A. Lotery, "Gene, cell and antibody-based therapies for the treatment of age-related macular degeneration," Biologics: Targets and Therapy, vol. 14, pp. 83-94, 2020.

[28] L. F. Hernandez-Zimbron, Z. A. Rubin, V. M. Raul et al., "Age-related macular degeneration: new paradigms for treatment and management of AMD," Oxidative Medicine and Cellular Longevity, vol. 2018, Article ID 8374647, 14 pages, 2018.

[29] E. Pawlowska, J. Szczepanska, A. Koskela, K. Kaarniranta, and J. Blasiak, "Dietary polyphenols in age-related macular degeneration: protection against oxidative stress and beyond," Oxidative Medicine and Cellular Longevity, vol. 2019, pp. 9682318-13, 2019.

[30] M. Hytti, N. Piippo, A. Salminen, P. Honkakoski, K. Kaarniranta, and A. Kauppinen, "Quercetin alleviates 4hydroxynonenal-induced cytotoxicity and inflammation in ARPE-19 cells," Experimental Eye Research, vol. 132, pp. 208-215, 2015.

[31] M. Hytti, D. Szabó, N. Piippo et al., "Two dietary polyphenols, fisetin and luteolin, reduce inflammation but augment DNA damage-induced toxicity in human RPE cells," The Journal of Nutritional Biochemistry, vol. 42, pp. 37-42, 2017.

[32] Q. Zhu, M. Liu, Y. He, and B. Yang, "Quercetin protect cigarette smoke extracts induced inflammation and apoptosis in RPE cells," Artificial Cells, Nanomedicine, and Biotechnology, vol. 47, no. 1, pp. 2010-2015, 2019.
[33] W. Du, Y. An, X. He, D. Zhang, and W. He, "Protection of kaempferol on oxidative stress-induced retinal pigment epithelial cell damage," Oxidative Medicine and Cellular Longevity, vol. 2018, Article ID 1610751, 14 pages, 2018.

[34] W. C. Huang, C.-J. Liou, S.-C. Shen, S. Hu, C.-Y. Hsiao, and S.-J. Wu, "Luteolin attenuates IL-1beta-induced THP-1 adhesion to ARPE-19 cells via suppression of NF-kappaB and MAPK pathways," Mediators of Inflammation, vol. 2020, Article ID 9421340, 15 pages, 2020.

[35] S.-Y. Lin, C.-L. Lin, C.-H. Chang, H.-C. Wu, C.-H. Lin, and C. H. Kao, "Risk of age-related macular degeneration in patients with prostate cancer: a nationwide, population-based cohort study," Annals of Oncology, vol. 28, no. 10, pp. 2575-2580, 2017.

[36] X. Wang, W. Ma, S. Han et al., "TGF-beta participates choroid neovascularization through Smad2/3-VEGF/TNF-alpha signaling in mice with Laser-induced wet age-related macular degeneration," Scientific Report, vol. 7, no. 1, p. 9672, 2017.

[37] X. Chen, R. Han, P. Hao et al., "Nepetin inhibits IL-1 $\beta$ induced inflammation via NF- $\kappa$ B and MAPKs signaling pathways in ARPE-19 cells," Biomedicine and Pharmacotherapy, vol. 101, pp. 87-93, 2018.

[38] R. S. Duncan, L. Rohowetz, A. Vogt, and P. Koulen, "Repeat exposure to polyinosinic:polycytidylic acid induces TLR3 expression via JAK-STAT signaling and synergistically potentiates NF $\kappa$ B-RelA signaling in ARPE-19 cells," Cellular Signalling, vol. 66, Article ID 109494, 2020.

[39] A. Lennikov, M. S. Saddala, A. Mukwaya, S. Tang, and H. Huang, "Autoimmune-mediated retinopathy in CXCR5deficient mice as the result of age-related macular degeneration associated proteins accumulation," Frontier in Immunology, vol. 10, p. 1903, 2019.

[40] X. Jin, C. Wang, W. Wu et al., "Cyanidin-3-glucoside alleviates 4-hydroxyhexenal-induced NLRP3 inflammasome activation via JNK-c-jun/AP-1 pathway in human retinal pigment epithelial cells," J Immunology Research, vol. 2018, Article ID 5604610, 8 pages, 2018.

[41] X. Gu, F.-f. Yuan, X. Huang et al., "Association of PIK3CG gene polymorphisms with attention-deficit/hyperactivity disorder: a case-control study," Progress in Neuro-Psychopharmacology and Biological Psychiatry, vol. 81, pp. 169-177, 2018.

[42] K. Totsuka, T. Ueta, T. Uchida et al., "Oxidative stress induces ferroptotic cell death in retinal pigment epithelial cells," Experimental Eye Research, vol. 181, pp. 316-324, 2019.

[43] D. Han, X. Wu, L. Liu et al., "Sodium tanshinone IIA sulfonate protects ARPE-19 cells against oxidative stress by inhibiting autophagy and apoptosis," Scientific Reports, vol. 8, no. 1, p. 15137, 2018. 